\title{
Las diferencias entre Colombia y Perú en la finalización del conflicto armado interno ${ }^{1}$
}

\author{
Paola Alexandra Sierra-Zamora ${ }^{2}$ \\ Universidad Católica de Colombia \\ Manuel Bermúdez-Tapia ${ }^{3}$ \\ Universidad Privada San Juan Bautista \\ Andrés Eduardo Fernández-Osorio ${ }^{4}$ \\ Escuela de Aviación del Ejército
}

1 Este capítulo hace parte de los resultados del proyecto de investigación "Desafíos contemporáneos para la protección de derechos humanos en escenarios de posconflicto desde enfoques interdisciplinarios" del Grupo de Investigación Persona, Instituciones y Exigencias de Justicia, del Centro de Investigaciones Sociojurídicas, Facultad de Derecho, de la Universidad Católica de Colombia, registrado con el código COL0120899 de Minciencias. También contó con la colaboración de los siguientes proyectos de investigación: "La legitimidad de las Fuerzas Militares en la geopolítica nacional e internacional de Colombia” del Grupo de Investigación en Ciencias Militares, de la Escuela Militar de Cadetes "General José María Córdova” (ESMIC), registrado con el código COL0082556 de Minciencias; y "Análisis del perfil criminológico de adolescentes infractores para evaluar tasas de reincidencia delictiva que afecte la seguridad ciudadana", aprobado por Resolución Vicerrectoral N. 0 058-2018-VRIUPSJB de la Universidad Privada San Juan Bautista, Perú. Los puntos de vista y los resultados de este artículo pertenecen a los autores y no reflejan necesariamente los de las instituciones participantes.

2 Abogada de la Universidad Católica de Colombia. Magíster en Derechos Humanos, Democracia y Justicia Internacional por la Universitat de València, España. PhD (c) del Programa de Derechos Humanos, Democracia y Justicia Internacional por la Universitat de València, España. Investigadora del Grupo de Investigación Persona, Instituciones y Exigencias de Justicia, de la Universidad Católica de Colombia. Orcid: https://orcid.org/0000-0002-3146-7418 - Contacto: pasierra@ucatolica.edu.co

3 Abogado (magna cum laude) de la Pontificia Universidad Católica del Perú. Profesorinvestigador de la Universidad Privada San Juan Bautista y profesor de la Facultad de Derecho de la Universidad Nacional Mayor de San Marcos. Consultor en temas de reforma del sistema de justicia en el Perú para el Banco Mundial, el Congreso de la República, el Poder Judicial y la Academia de la Magistratura. Orcid: https://orcid.org/0000-0003-1576-9464 - Contacto: manuel.bermudez@upsjb.edu.pe

4 Teniente Coronel del Ejército Nacional de Colombia. PhD en Derecho y Ciencia Política, de la Universidad de Barcelona, España. Magíster en Economía, Estado y Sociedad: Política y Seguridad, University College London, Reino Unido. Magíster en Relaciones Internacionales, Higher School of Economics, Moscow, Federación de Rusia. Investigador del Grupo de Investigación en Ciencias Militares de la Escuela Militar de Cadetes "General José María Córdova” y del Grupo de Investigación en Aviación Militar de la Escuela de Aviación del Ejército. Orcid: https://orcid.org/0000-0003-0643-0258 - Contacto: andres.fernandez@buzonejercito.mil.co 


\section{Introducción}

Cuando se evalúa la realidad social peruana contemporánea, especialmente entre los ańos 2010 y 2019, se observa un contexto de violencia desmedida en casos específicos: en el ámbito de las relaciones de pareja, en las familias en crisis, en la exclusión social, el racismo, la discriminación, el bullying y otras manifestaciones. Sin embargo, en el ámbito del diseño de políticas públicas (Zornoza et al., 2009) y en la doctrina, solo se ha tomado en cuenta el contexto de la violencia de género, distinta a la violencia generada en el ámbito de las relaciones de pareja y en el interior de una familia en crisis. Este error conceptual permite plantear un análisis mucho más profundo sobre las causas de la violencia social en el Perú, para plantear una solución alternativa. En este punto, se hace referencia al ámbito colombiano, porque es posible identificar una serie de situaciones similares, en mayor o menor medida. La razón de este estudio comparativo es que en Colombia se registra la siguiente información:

a. Ha tenido un proceso de violencia interna con un conflicto armado de más de cincuenta años

b. Registra un alto nivel de ilegitimidad institucional en las diferentes entidades estatales

c. Registra un elevado nivel de corrupción entre sus autoridades políticas

d. Registra un presidente acusado de cometer delitos de lesa humanidad

e. Registra procesos en trámite ante la Corte Interamericana de Derechos Humanos por la violación de derechos de sus ciudadanos

f. Registra una guerrilla (disminuida y focalizada en una zona territorial muy limitada) que plantea la continuidad de un conflicto contra el Estado

g. Registra un contexto en el cual el narcotráfico ha intervenido en las altas esferas del gobierno

Estos elementos están muy próximos a la realidad peruana y, por tanto, permiten un análisis comparativo, especialmente, en un sentido prospectivo, complementado con un estudio cualitativo y transversal que procura analizar 
la realidad sociopolítica para desarrollar las diferencias implicadas por la ejecución de una Justicia Transicional, la cual no se generó en el Perú.

\section{Un problema en común de Colombia y Perú}

En Colombia, a raíz del Acuerdo de Paz (2016) entre el Gobierno nacional y las Fuerzas Armadas Revolucionarias de Colombia-Ejército del Pueblo (en adelante, FARC-EP), se viene planificado poner en ejecución la Justicia Transicional. Esta última tendría por objetivo máximo la atención de la finalización del conflicto armado interno que ha generado una profunda crisis en Colombia, pues está presente en la cuarta parte de su historia republicana.

El contexto comparativo de Colombia resulta ideal para evaluar una aproximación a este mecanismo procesal, en particular, si se toma en cuenta que Perú también ha pasado por un proceso de conflicto armado interno debido a las acciones terroristas de Sendero Luminoso (SL) y el Movimiento Revolucionario Túpac Amaru (MRTA). De ambos grupos se llegó a tener conocimiento sobre sus relaciones con organizaciones criminales de traficantes de droga, hecho que ha mutado en la actualidad como "narcoterrorismo", especialmente en el Valle de los ríos Apurímac, Ene y Mantaro — VRAEM(Van Dun, 2009).

Semejante realidad tan idéntica a la de Colombia, donde el terrorismo y el narcotráfico son el común denominador, permite detallar por qué el Gobierno peruano optó en un caso por reconocer el conflicto armado interno, mientras que en el otro lo negó. Surge, entonces, la necesidad de plantear, como duda metodológica, la pregunta de cuáles fueron las razones por las que en el Perú solo se alcanzó a tener una Comisión de la Verdad y no un proceso que involucrara la institucionalización de una Justicia Transicional. De haberse instaurado este último, se hubiera evitado un contexto de violencia social agudo, como el que actualmente se registra en la sociedad colombiana.

Conviene aclarar en este momento que las FARC-EP, en una mínima facción, han anunciado la continuidad del conflicto armado interno, hecho que cuenta con el apoyo institucional del Gobierno de Venezuela, pese a que esta guerrilla ya tiene el registro formal y objetivo de tener vínculos con grupos 
de narcotraficantes. De forma similar, en el Perú, Sendero Luminoso aún mantiene una mínima organización y periódicamente ejecuta acciones contra las Fuerzas Militares, especialmente en la zona del VRAEM.

Como se puede observar, las similitudes y diferencias parten del modo en el cual cada país optó por evaluar el conflicto armado interno. Esto responde sobre todo a una decisión política frente a una situación crítica que involucraba una acción insurgente y de rebeldía que afectaba una porción importante del territorio nacional.

\section{Las consecuencias materiales de un conflicto armado interno}

Explicar la realidad nacional tanto peruana como colombiana, en función de una validación de postulados teóricos resulta complicado, porque se asume que los hechos ocurridos en cada país son esquivos a un análisis comparativo. Una afirmación que contiene un error metodológico, por cuanto las diferencias son muchas, pero las similitudes superan los elementos contradictorios.

En el ámbito comparado, surge una serie de elementos importantes a analizar respecto del modo de comprender la violencia como problema social frente a la legitimidad del Estado. En efecto, este último está llamado a afrontar esta realidad para resolverla, con el doble fin de proteger la sociedad y de legitimar su poder sobre la comunidad. Los elementos a analizar son los siguientes:

a. Aparición de grupos de guerrillas que han optado por luchar contra el Estado o contra el Gobierno nacional. El contenido político o ideológico que aducen estos grupos para acudir a la lucha insurgente hace que estas guerrillas sean muy similares tanto en Colombia como en Perú.

b. Las guerrillas han optado por capturar el poder y ejecutar una acción de cambio de la política nacional. Tanto en Colombia como en Perú, las guerrillas han planteado acceder al poder de modo violento y no a través de la democracia, esencialmente porque han asumido una "representación" que los legitima en función de una relación con la población históricamente afectada en determinados territorios. 
En Colombia, esto identifica a las FARC-EP con la "República de Marquetalia” y a Sendero Luminoso con la zona de Ayacucho como región de incidencia y expansión de sus actos de terror.

c. Las acciones de insurgencia han involucrado actos de lucha militar con armas de combate y confrontación directa con las Fuerzas Armadas. En Colombia, estos actos fueron categorizados como conflicto armado interno, pero, en esencia, guardan una similitud con los actos de terrorismo ejecutados por las guerrillas en el Perú (Cubides-Cárdenas et al., 2019). Sin embargo, en este contexto, las guerrillas a nivel general han optado por aceptar las reglas del derecho internacional humanitario a su favor, pero, cuando han ejecutado actos de terrorismo (atentados contra la población), la fundamentación de sus actos no es válida, pues estos están igualmente prohibidos por el derecho internacional humanitario.

Se trata, entonces, de un contexto muy particular y especial, porque implica un proceso de violencia extrema con uso de armamento que puede dar paso a una guerra civil y que, por ello, requiere de una terminología apropiada: conflicto armado interno. En este punto, los Estados, a través de sus gobiernos nacionales, han tenido que ejecutar acciones de tutela de sus propios intereses, que se enuncian en orden de prelación:

a. Defensa de la población afectada, tanto en forma directa como indirecta.

b. Defensa de las instituciones del Estado, a todo nivel y en todo el territorio nacional.

c. Defensa de la democracia en el ámbito interno, por cuanto el sostenimiento del país debía ser garantizado.

d. Protección de las actividades en el ámbito interno del país, por cuanto la productividad no podía ser condicionada por el conflicto armado interno.

Estos intereses aluden al propio origen de los Estados, sobre la base de la vinculación de una necesidad a ser atendida: proteger la población ante las 
acciones de pueblos externos y evitar situaciones de violencia en la comunidad que reside en un territorio determinado, población que requiere de una administración pública para que la dirija.

Téngase presente, en este contexto, que surgen automáticamente las referencias a las teorías del Estado, del derecho constitucional y del derecho internacional, de acuerdo con las cuales el pueblo, la comunidad, el territorio, la administración pública, la legitimidad y la gobernabilidad son una constante en la historia universal. Por tanto, resulta interesante analizar estas referencias comparadas y, así, plantear una de las preguntas clave de esta investigación: ¿Por qué en el Perú no se aplicó una justicia transicional en el posterrorismo?

\section{El cambio de paradigmas: el conflicto armado interno frente a los conflictos tradicionales}

Desde el final de la II Guerra Mundial, el mundo se ha desarrollado en función de nuevos estándares en los que se han modificado muchas variables teóricas (Hartel, 2011). Una de las más importantes es la evolución de los conflictos internacionales a los conflictos armados no internacionales o internos. No es que cada elemento sea excluyente o eventualmente antagónico, pero la generalidad es que los conflictos internacionales han disminuido al nivel de ser casi excepcionales y los conflictos armados no internacionales o internos se han incrementado en modo notorio en todos los hemisferios.

Los cambios en las formas de establecer las relaciones internacionales, en las que prima la diplomacia y la coerción económica frente al poder militar, cede a un nuevo panorama en el ámbito de la evaluación de comportamientos estatales respecto de la intervención militar en otras latitudes. La necesidad de salvaguardar la seguridad nacional y el orden pacífico en la comunidad nacional constituía una prioridad para el Estado, una vez culminada la Segunda Guerra, porque con ello se legitimaba ante la población, debido al contexto de violencia extrema que empezó a generarse por más de cinco décadas. En el ámbito social, la búsqueda de la paz constituye una esperanza para la propia nación peruana (como población), puesto que una fracción de su historia republicana se había visto condicionada a una convivencia con la violencia provocada por 
grupos insurgentes y contrarios al Estado, como ha sucedido en España, Italia, Inglaterra y Colombia, situación que permite el análisis comparativo.

De esta forma, es posible analizar cómo se desarrolla la búsqueda de la paz, principalmente a través de acuerdos del Gobierno con fracciones poblacionales, cuya característica es de beligerancia y confrontación sin ánimo de provocar un proceso separatista del territorio, como sucedió por ejemplo con el Ejército Republicano Irlandés (IRA) o el Euskadi Ta Askatasuna (ETA) en España.

\section{Colombia y Perú ante el enemigo interno: semejanzas y diferencias}

Para mayor claridad en la exposición de los fundamentos del presente texto, se llevan a cabo las comparaciones siguientes:

a. Los niveles de violencia política generados por grupos insurgentes, el terrorismo contra el Estado, el conflicto armado interno y todas sus variables complementarias y conexas, tanto en Colombia como en Perú, dieron pie a dos problemas.

i. La lucha armada de las Fuerzas Militares (Colombia) y las Fuerzas Armadas (Perú) contra los grupos alzados en armas.

ii.. La lucha vinculada a un contexto de intervención o presión internacional.

A diferencia de Perú, Colombia sí ha tenido un nivel de presión internacional en la lucha contra los grupos insurgentes. Este panorama cambió a raíz de las violaciones de los DD. HH. con el gobierno de Alan García (Caso El Frontón, Cayara), que pasaron inadvertidas inicialmente, hasta que el 16 de agosto del 2000 se emitió la Sentencia de la CIDH en el caso Durand y Ugarte vs. Perú, en la que se acreditó la violación de DD. HH. por parte del Gobierno de turno.

Posteriormente, los fallos de la CIDH contra Perú han modificado el panorama de tutela de DD. HH. en todo el mundo, principalmente por los aspectos procesales y sustantivos emitidos. 
b. El surgimiento de una vinculación paulatina con el narcotráfico.

Tanto en Colombia como en Perú, todos los grupos terroristas se vincularon con el narcotráfico, porque así garantizaban varios elementos:

i. El control de un territorio, gracias a lo cual podían dominar la producción o movilización de droga para el mercado internacional.

ii. El suministro de recursos económicos que les garantizan sostenibilidad.

iii. La vinculación con elementos de la administración pública para el acceso a información privilegiada que les garantice un statu quo, con lo cual se da lugar a un mecanismo de corrupción "institucionalizado" por el propio Estado.

Tabla 1. Síntesis comparativa entre los conflictos internos de Colombia y Perú

\begin{tabular}{|c|c|c|}
\hline Gobiernos de Colombia & Terrorismo & Gobiernos de Perú \\
\hline $\begin{array}{l}\text { Gobiernos en } \\
\text { confrontación directa } \\
(1964-1982)\end{array}$ & \multirow{5}{*}{$\begin{array}{c}\text { Guerrillas de Colombia } \\
\text { en orden cronológico: } \\
\text { FARC (1964) } \\
\text { ELN (1964) } \\
\text { EPL (1967) } \\
\text { M19 (1970) } \\
\text { Guerrillas de Perú } \\
\text { en orden cronológico } \\
\text { Sendero Luminoso } \\
\text { (1980) } \\
\text { MRTA (1984) } \\
\text { Comando } \\
\text { Rodrigo Franco } \\
\text { (entre 1985-1986) }\end{array}$} & $\begin{array}{l}\text { Gobiernos en } \\
\text { confrontación directa } \\
(1980-1993)\end{array}$ \\
\hline $\begin{array}{l}\text { Belisario Betancur Cuartas } \\
(1982-1986)\end{array}$ & & $\begin{array}{l}\text { Fernando Belaúnde Terry } \\
(1980-1985)\end{array}$ \\
\hline $\begin{array}{l}\text { Virgilio Barco Vargas } \\
(1986-1990)\end{array}$ & & $\begin{array}{l}\text { Alan García Pérez } \\
(1985-1990)\end{array}$ \\
\hline $\begin{array}{l}\text { César Gaviria Trujillo } \\
(1990-1994)\end{array}$ & & $\begin{array}{l}\text { Alberto Fujimori } \\
\text { (1990-1992 Captura } \\
\text { de Abimael Guzmán) }\end{array}$ \\
\hline $\begin{array}{l}\text { Ernesto Samper Pizano } \\
(1994-1998)\end{array}$ & & $\begin{array}{l}\text { Alberto Fujimori } \\
(1993-2001) \\
\text { CIDH, Sentencia } \\
\text { Caso Castillo Petruzzi } \\
(30 / 05 / 1999) \\
\text { CIDH, Sentencia } \\
\text { Caso Barrios Altos } \\
(14 / 03 / 2001)\end{array}$ \\
\hline
\end{tabular}




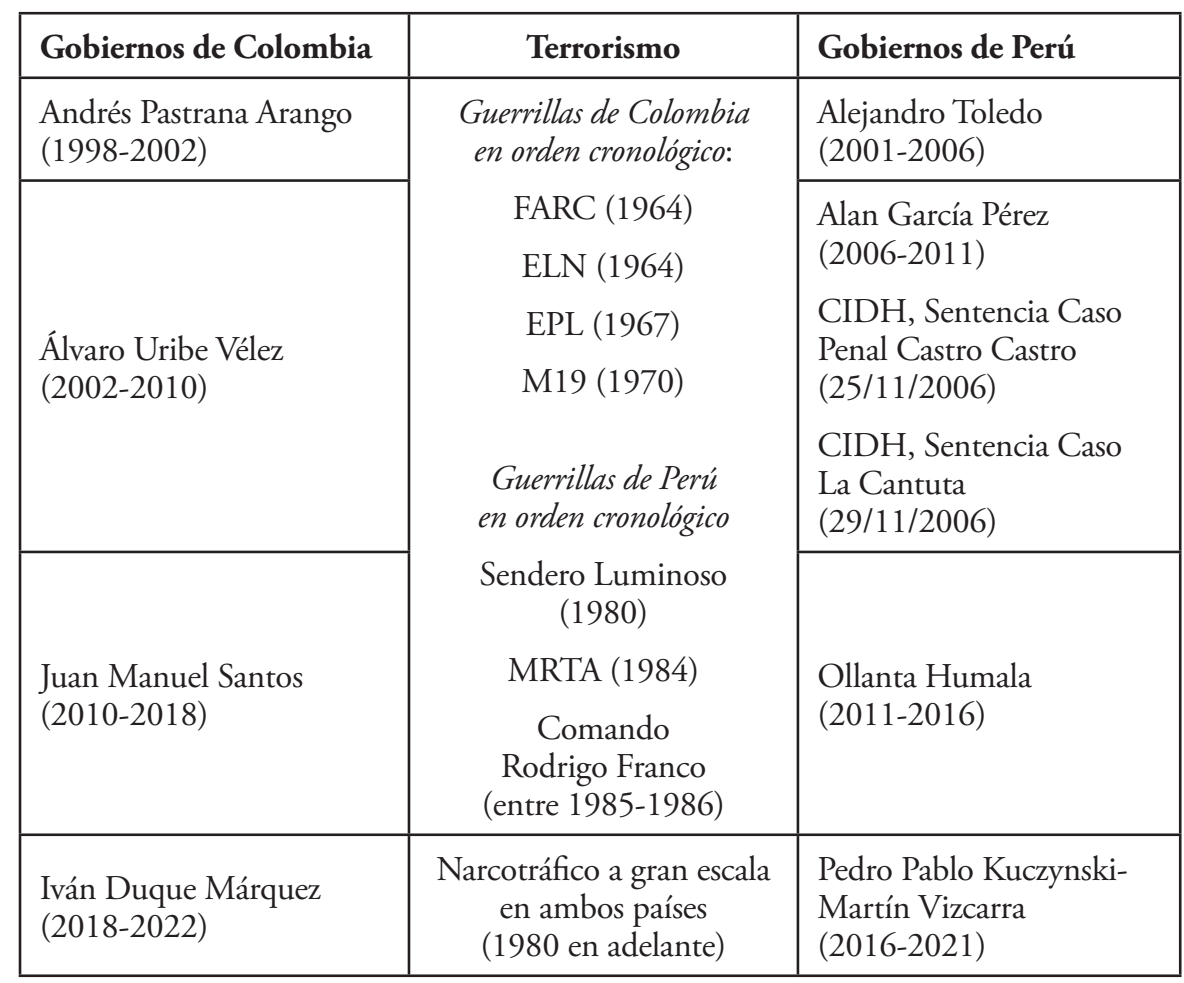

Fuente: elaboración propia

Como resultado de esta evaluación comparativa, solo Colombia desarrolló una acción oficial, formal y de Estado frente a la lucha armada interna, con el fin de negociar el acceso a la paz social con grupos insurgentes a través de una concertación política. Con ello se logró el ingreso a la vida política del Movimiento 19 de Abril (M19) y de las FARC-EP, que actualmente participan en elecciones democráticas y populares. En el Perú, solo se registró una Comisión de la Verdad, pero esto tiene una explicación, sobre las bases siguientes:

a. Con la captura de Abimael Guzmán (1992) y de Víctor Polay Campos (1992), se desarticuló la cúpula de los dos grupos terroristas más importantes.

b. La falta de una organización y sostenibilidad armada en el tiempo propició que las huestes terroristas se vieran limitadas en zonas específicas, como actualmente ocurre en el VRAEM, en Ayacucho. 
c. La legitimidad de los grupos terroristas era mínima, principalmente, por los niveles de violencia y ejecución de actividades delincuenciales comunes llevados a cabo.

La diferencia entre Colombia y Perú marca un elemento trascendente en esta evaluación, por lo cual es necesario detallar que el punto de quiebre es el proceso de democratización en la búsqueda de una solución pacífica y no a través de las armas. Sin embargo, conviene tener en cuenta que la paz en ambos países aún no se ha consolidado y este sigue siendo un factor vinculado a un nuevo fenómeno social: las guerrillas, la corrupción y el narcotráfico, por tanto la concepción de la "lucha" contra este nuevo flagelo social varía y se deben considerar dos opciones:

a. La construcción de una política de Estado en concordancia con lo determinado por la Constitución, con políticas públicas específicas y complementarias entre sí.

b. El diseño de políticas públicas en el ámbito punitivo, productivo y de asistencia social.

En Colombia, se diseñó la acción del Gobierno nacional con base en el primer punto, mientras que en el Perú se diseñó sobre la base de las acciones de la segunda referencia.

\section{Las soluciones legítimas de acuerdo con la Constitución}

Tomando en cuenta que en Colombia el conflicto armado interno tomó más de cincuenta años y en el Perú un promedio de trece años, los Estados debían asumir el contexto de la etapa posterrorista, porque los niveles de violencia habían afectado sobremanera a la población y se tenía que ejecutar una acción programática y sostenible en el tiempo para que así la paz alcanzara a tener una legitimidad social (Habermas, 1992). Colombia pensó en esta situación, pero en el Perú no se tomó en cuenta el contexto de violencia social propio del terrorismo, por ello los resultados son diferentes. En el Perú, en 
efecto, no se tomó en cuenta el contexto de violencia progresiva provocada por los factores siguientes:

a. El desarrollo de las migraciones y el impacto social en el ámbito de las relaciones poblacionales en las grandes ciudades, factor que provocó la amplificación de los niveles de discriminación y racismo en el país.

b. El contexto económico negativo provocado por la Reforma Agraria de Juan Velasco Alvarado (1969), que generó una mayor ola de migración de campesinos e indígenas a las zonas urbanas, con el consiguiente quiebre de la economía nacional.

c. La crisis del Estado en lo financiero y a nivel de gestión pública, que provocó que los gobiernos de Francisco Morales Bermúdez (19751980) y de Alberto Fujimori (1990-1995) se vieran sumergidos en el déficit económico que propició el paulatino nivel de negligencia y de corrupción en el país que alcanzó un nivel de institucionalidad desde 1985 hasta la fecha.

d. El impacto del terrorismo que generó una movilidad social que nunca se reconoció como desplazamiento forzado, porque sociológicamente no se admitió el conflicto armado interno. Un detalle de suma importancia que afectó la propia naturaleza de las relaciones familiares hasta provocar nuevas categorías de familia, como la familia separada, la familia dividida, la familia monoparental, la familia amplia y la familia ensamblada, así como la generación de parejas en convivencia de mala fe, convivencias paralelas y relaciones concubinales, todas ellas con patrones excluyentes entre sí.

Este es un conjunto de situaciones muy particulares que diferencian la realidad de Perú respecto de la de Colombia, en la que solo los niveles de violencia insurgente y narcotráfico pueden provocar un panorama equivalente. Por ello, la diferenciación de una politica de Estado frente a una politica pública, diferenciación que es vital para entender los comportamientos de los Gobiernos nacionales evaluados. 
Una política de Estado incluye la reforma del Estado para generar legitimidad en la búsqueda de la paz ante una situación de violencia extrema, situación que fue reconocida en Colombia. Una política de Estado implica limitar un problema social en función de una atención o acción directa del Estado para atender requerimientos sociales que son temporales o específicos.

Así, en el Perú se ejecutó una negativa visión de la realidad social con una proyección en el tiempo, factor que ha propiciado la generación de un contexto contemporáneo en el que prima la corrupción en la administración pública y la violencia social en la comunidad.

\section{La Justicia Transicional como una alternativa en Colombia}

Con la comprensión de que se requiere una legitimidad social, Colombia modificó su Constitución (Cubides-Cárdenas et al., 2019) y sometió a referéndum el Acuerdo de Paz. Si bien los resultados fueron negativos, es de observarse que igual el Gobierno nacional insistió en su proyecto y al final está alcanzando relativo éxito. Sin embargo, para alcanzar esto se ha debido ejecutar en forma progresiva lo siguiente:

a. Reformar la Constitución Política con el Acto Legislativo 01 de 2012 (Suárez López \& Jaramillo Ruiz, 2014).

b. La facilitación de los mecanismos estatales y de negociación que permitan finalizar el conflicto interno con las FARC-EP.

c. La generación de mecanismos jurisdiccionales excepcionales: Justicia Transicional.

d. El tratamiento normativo, jurisdiccional y procesal diferenciado de los distintos grupos armados al margen de la ley, para detallar un nivel de participación en el conflicto armado interno y establecer responsabilidades específicas.

e. La regulación de un mecanismo estatal que permita garantizar las obligaciones del Estado para la investigación y sanción de actos contrarios a los derechos humanos de los ciudadanos colombianos. 
f. La estructura de un proceso social y político que permita materializar la paz en un plazo relativamente breve, como política pública.

g. La creación de una Comisión de la Verdad.

h. La generación de un mecanismo de priorización de atención de casos y situaciones, para así atender de forma particular y especial temas específicos.

En suma, un conjunto de mecanismos programáticos y articulados entre sí para materializar un objetivo nacional, pero que sin embargo generaba algunos inconvenientes, principalmente, en cuanto a su propia "naturaleza jurídica” y por ello es que se planteó, casi en forma inmediata, un proceso de inconstitucionalidad que fue definido por la Corte Constitucional en la Sentencia C-579 de 2013. En dicha sentencia se establecen las pautas para la "constitucionalización de mecanismos de Justicia Transicional", concretamente en la aplicación de criterios de selección y priorización en la judicialización de crímenes internacionales (Ambos \& Zuluaga, 2014).

Como resultado de esta situación, se plantean dos elementos vinculantes para todo el proceso de materialización de la paz: la determinación del deber estatal de garantizar los derechos humanos y el juzgamiento de todas las violaciones e infracciones graves al derecho internacional humanitario cometidas en Colombia (Silva \& Cleves, 2014). Bajo estas premisas, la necesidad de evitar situaciones de impunidad se establece como un objetivo casi prioritario para Colombia, de conformidad con su propia legislación y en función de sus compromisos internacionales.

Complementariamente está la necesidad de salvaguardar los derechos de las víctimas (Sierra-Zamora, 2018), lo que provocará una serie de condiciones y situaciones muy complicadas de evaluar, tomando en cuenta el propio marco normativo y sustantivo aplicable a la evaluación del derecho internacional humanitario y el derecho internacional de los derechos humanos. Premisas que finalmente son orientadas por la Corte Constitucional colombiana para permitir la legalidad de la Justicia Transicional y que se pueda operar el tránsito a la paz. Un panorama no observado en el Perú, porque se consideró que la finalización de la lucha contra el terrorismo generaría de forma automática una paz social. 


\section{El caso peruano y su etapa de posterrorismo}

Tomando en cuenta lo expuesto, y con base en la experiencia colombiana respecto de la realidad peruana, es necesario evaluar los aspectos siguientes:

a. ¿La búsqueda de la paz se debía ejecutar a cualquier costo? El acceso a la Paz como mecanismo político en Colombia no está teniendo el nivel de legitimidad social que debería tener, porque surgen niveles de oposición que se basan en la evaluación diferenciada de acciones contrarias a la sociedad y al propio Estado. En consecuencia, quien en inicio era considerado guerrillero tiene ahora la calidad de un representante ante un ente político nacional y no puede ser acusado de ningún delito, mientras que un miembro de las Fuerzas Militares, que actuó en cumplimiento de mandatos en defensa de su país, puede ser acusado de haber cometido un delito de lesa humanidad. En el Perú, esta situación no se ha producido, y actualmente hay un rechazo tanto social como estatal a toda legitimación de actividades ejecutadas por grupos afines a Sendero Luminoso y el Movimiento Revolucionario Túpac Amaru. Sin embargo, las acciones de estas organizaciones terroristas se han mantenido tanto a través de acciones de sus simpatizantes en el extranjero (a nivel ideológico) como en el país a través de acciones de terrorismo contra las Fuerzas Armadas, sobre todo en el VRAEM.

b. ¿La identificación de las víctimas se debe hacer solo desde una perspectiva? Conforme se han dado las sentencias de la CIDH contra Perú, es posible observar que las víctimas solo han sido las partes procesales y en ningún caso se ha tomado en cuenta el daño directo a la población peruana afectada. Un detalle que no puede dejar de señalarse, porque parecería que solo el Estado ha ejecutado actos contrarios a los DD. HH.

c. ¿Los procedimientos jurisdiccionales pueden ser derivados o modificados a toda costa? Nótese que en el Perú se ejecutó una reforma normativa a la legislación contra el terrorismo, merced a la intervención de la CIDH. En Colombia, este debate está provocando la crisis 
de conceptos teóricos como el del juez natural, un detalle de suma importancia en el ámbito procesal.

\section{Conclusiones}

El registro de niveles de violencia social extremos en el Perú del nuevo milenio plantea como cuestión metodológica determinar las razones por las cuales el Estado no se preparó para la etapa posterior a la lucha contra el terrorismo.

La posibilidad de hacer un análisis comparado entre esa realidad política y social y la de Colombia tomó ofrece un amplio campo de estudio. Por ejemplo, Perú, a diferencia de Colombia, no ejecutó una acción con un alcance de legitimidad superior y centró su acción en las investigaciones e informes de la Comisión de la Verdad y otras acciones derivadas, porque asumió que la violencia política y social provocada por el terrorismo (y el narcotráfico) era un problema puntual, que podía resolverse apenas con intervención militar. Un error que se aprecia en la actualidad y se acredita en los casos de feminicidio que llegan a niveles inaceptables.

\section{Referencias}

Ambos, K., \& Zuluaga, J. (2014). Justicia de Transición y Constitución. Análisis de la sentencia C-579 de 2013 sobre el Marco Jurídico para la Paz. Justicia de Transición y Constitución. Temis.

Cubides-Cárdenas, J. A., Sierra-Zamora, P. A., Ortiz, D. A., \& Caballero, N. P. (2019) Terrorism by the FARC-EP and public policies oriented towards national security in Colombia during 1990-2000. Revista Cientifica General José María Córdova, 17(26), 309-325. http://dx.doi.org/10.21830/19006586.404

Habermas, J. (1992). Teoría de la acción comunicativa I. Racionalidad de la acción y racionalidad social. Taurus.

Hartel, M. (2011). El juicio de Nuremberg como antecedente de la Corte Penal Internacional desde las tres dimensiones del mundo jurídico. Revista de Integración y Cooperación Internacional, 7, 5-10.

Sierra-Zamora P. A. (2018). La tutela de los derechos humanos en situaciones de postguerra: el caso colombiano [Documento de trabajo]. OSF Home. https://doi.org/10.17605/OSF. IO/7KM83 
Silva, P., \& Cleves, G. (2014). La jurisprudencia constitucional colombiana en el año 2013: el control de constitucionalidad por sustitución y el amparo reforzado a los sujetos de especial protección constitucional. Anuario Iberoamericano de Justicia Constitucional, 18, 587-620.

Suárez López, B., \& Jaramillo Ruiz, F. (2014). La satisfacción del derecho a la justicia en el marco del proceso de paz colombiano. Una mirada a la evolución en materia de responsabilidad penal en el contexto de un proceso de paz y de los actuales estándares internacionales. Estudios Socio-Jurídicos, 16(2), 61-88. dx.doi.org/10.12804/esj16.02.2014.02

Van Dun, M. (2009). Cocaleros: violence, drugs, and social mobilization in the post-conflict upper Huallaga Valley, Perú. Rozenberg.

Zornoza, J., Arroyave, S., \& Rodríguez, N. (2009). Políticas públicas en sistemas críticos: el caso latinoamericano. Universidad Nacional de Colombia. 